\title{
A Novel Variant of SMARCB1 in Rare Familial Schwannomatosis Identified by Whole-Exome Sequencing and Genotype-Phenotype Correlation Analysis.
}

\section{Conghui Wang}

The First Affiliated Hospital of Zhengzhou University

\section{Yuqiong Chai}

Luoyang Maternal and Child Health Care Hospital

\section{Xuechao Zhao}

The First Affiliated Hospital of Zhengzhou University

\section{Qianqian Li}

The First Affiliated Hospital of Zhengzhou University

\section{Chen Chen}

The First Affiliated Hospital of Zhengzhou University

Xiangdong Kong ( $\sim$ kxd1968@163.com )

The First Affiliated Hospital of Zhengzhou University

\section{Research Article}

Keywords: Novel variants, Schwannomatosis, SMARCB1, WES

Posted Date: December 15th, 2021

DOI: https://doi.org/10.21203/rs.3.rs-1157679/v1

License: (c) (i) This work is licensed under a Creative Commons Attribution 4.0 International License.

Read Full License 


\section{Abstract}

Background: Variants in the tumor suppressor gene SMARCB1 could cause different conditions. In some cases, germline and somatic variants in SMARCB1 are implemented in schwannomatosis. But the genotype and phenotype correlation for variants in SMARCB1 has not been determined.

Methods: A Chinese schwannomatosis family with an autosomal dominant inheritance pattern was recruited. Whole-exome sequencing (WES) was performed to discover the causative variant, followed by Sanger sequencing. We evaluated the Human Gene Mutation Database (HGMD) regarding SMARCB1 variants and validated associated phenotype records to assess phenotype-genotype relationships.

Results: A novel deletion variant c.885_896delGAAGCTGTGCTC p.(295_299del】 in SMARCB1 was identified in the affected family members and cosegregated with phenotypes in the pedigree. About $51.1 \%$ of variants in SMARCB 1 located in Snf5 subunit, $80.7 \%$ of variants were loss-of-function (LOF) variants, and more variants located in the Snf5 subunit of SMARCB1 in Rhabdoid tumour (67.8\%) than that in schwannomatosis (25.7\%).

Conclusions: Our study expands the variant spectrum of SMARCB1 and the genetic background of schwannomatosis, confirms the clinical indications for genetic screening of the SMARCB1 gene, and has implications for genetic counseling in this disease.

\section{Background}

Schwannomatosis (MIM 162091) is a rare disorder characterized by the predisposition to develop benign multiple non-vesticular schwannomas and, in some cases, meningiomas and so on [1-3]. Examination and diagnosis of schwannomatosis has been hampered by the rarity of familial cases and the difficulty in separating schwannomatosis from NF2 cases [4]. Schwannomatosis is very rare, having an incidence of 0.58 cases/ 1 million persons every year [5]. Familial and sporadic schwannomatosis accounts for about $15 \%$ and $85 \%$ of reported cases, separately [6]. SMARCB1, a tumor suppressor gene, is involved in very low proportion of schwannomatosis patients: about $50 \%$ of the familial cases in autosomal dominant fashion with incomplete penetrance, but no more than $10 \%$ of the sporadic cases [7]. Schwannomatosis shares clinical symptoms and it can be challenging to distinguish with neurofibromatosis type 2 (NF2), particularly in cases of mosaic conditions [8]. SMARCB1/INI1 is one of the core subunit of the ATP-dependent SWI/SNF chromatin remodeling complex, located at chromosomal position 22q11.2 [9]. So far, only 135 SMARCB1 variants have been reported in the Human Gene Mutation Database (Professional 2021.2, http://www.hgmd.org). Variants in SMARCB1 can cause different phenotypes, and the exact genotype-phenotype correlation for the variants in SMARCB1 need to be further clarified [10]. In this study, we revealed a novel variant c.885_896delGAAGCTGTGCTC (p.(296_299del)) (NM_003073.5) in SMARCB1 in a Chinese family with schwannomatosis.

\section{Patient Data And Methods}




\section{Subjects and clinical evaluation}

We observed a schwannomas family with autosomal dominant inheritance in our study. The diagnosis of schwannomas followed the consensus criteria of the National Institutes of Health, and the proband was confirmed with schwannomas by excisional biopsy and magnetic resonance imaging (MRI). Written informed consent was obtained from all participants, and this study was approved by the Medical Ethics Committee of the First Affiliated Hospital of Zhengzhou University. All procedures were carried out in accordance with the Declaration of Helsinki.

\section{Variant screening and analysis}

DNA was extracted from blood samples according to standard methods using commercially available kits (QIAGEN, Venlo, The Netherlands). Whole-exome sequencing was based on the illumina sequencing platforms. The sequence reads were aligned to the human reference genome reference obtained from the UCSC database version hg19 (http://genome.ucsc.edu). The mean sequencing depth was $104.52 \times$, and $98.23 \%$ of the targeted exome with $>20 x$ sequencing depth was obtained to accurately determine variants. Sequence variant interpretation was according to the American College of Medical Genetics and Genomics (ACMG) guidelines.

\section{Results}

\section{Clinical Manifestation}

Three patients of the family were clinically diagnosed with schwannomas (Fig. 1A). The proband (III1), a 27-year-old woman, was born normally. Lumbar pain occurred during rope skipping at the age of 18 years. The symptoms were gradually aggravated after stopping activities, and the night pain was the most serious. The proband developed a mass the size of sour jujube in the right neck half a month before admission at the age of 20 years. Pain and numbness were manifested in the right upper limb after extrusion. The tumor in the superior trunk of the right brachial plexus was diagnosed after operation. The pathological results showed (right supraclavicular fossa) schwannoma. After a year (at the age of 21 years), because of the occupied spinal canal space, the proband was admitted to the hospital. Intraspinal schwannoma was diagnosed during the operation, and the pathological results showed the schwannoma in the thoracic 1, 2 spinal canal. At the age of 24 years, the mass was found on the posterior thigh, the occupied left sciatic nerve was diagnosed during the operation. and the pathological results suggested the schwannoma of the left lower limb. Two years later (at the age of 26 years), pains without obvious cause occurred in the left upper limb. She was admitted to hospital with multiple skin lesions with nodules (Fig. 1B) and cervical intraspinal space occupying lesions. After operation, the operative and pathological findings and MRI results demonstrated schwannomas (intracranial, cervical intraspinal, thoracic intraspinal space occupying) (Fig. 2A-D).

The proband ( $\ 1)$, a 48-year-old man, was diagnosed with L4L5 intraspinal schwannoma and multiple schwannoma within both thighs (Fig. 2E-F). 
The late proband $(\mathbb{\nabla} 1)$, was dictated as schwannoma by clinical symptom descriptions without definitive diagnosis, and died at the age of 69 .

\section{Variant analysis of SMARCB1}

Whole-exome sequencing and Sanger sequencing revealed the heterozygous variant of the affected family members c.885_896delGAAGCTGTGCTC (p.(296_299del)) in SMARCB1 (Fig. 1C). The variant in $S M A R C B 1$ was absent in the healthy family members, suggesting the variant cosegregated with the phenotype in this family (Fig. 1A). Multiple sequence alignment of 5 different species indicated the conservation around the 296_299 residues within the Snf 5 subunit of the SMARCB1 (Fig. S1). According to $A C M G$, the variant of $S M A R C B 1$ was predicted to be likely pathogenic (PM1+PM2+PP1+PP3). Moreover, the variant was novel because it did not present in dbSNP, HGMD database, nor had been previously reported.

\section{Discussion}

In this Chinese pedigree, a novel and rare deletion variant (c.885_896delGAAGCTGTGCTC p. (296_299del)) in exon 7 was identified, which results in a truncated protein of 4 amino acid residues instead of full-length INI protein.

SMARCB1 mutated familial schwannomatosis transmitted in autosomal dominant fashion with incomplete penetrance accounts for about 7-8\% of reported cases. In our study, the variant (c.885_896delGAAGCTGTGCTC p.(296_299del)) in SMARCB1 has been shown to segregate with disease in the family members in 2 generations. Multiple sequence alignment of 5 different species reveals a high degree of conservation within the Snf5 subunit, implying its functional importance and the potential pathogenicity of the variant. The novel variant is a likely disease-causing loss of function variant according to the ACMG classification guidelines.

It is interesting that variants in SMARCB1 can cause two very different phenotypes: schwannomatosis or malignant rhabdoid tumours (MRTs), and it may depends on the the variant types and location within the domain. Nontruncating (missense or splicing) variants are the major inherited variants found in familial schwannomatosis. Moreover, a higher proportion of truncating variants were found in sporadic schwannomatosis patients. The most common alteration identified so far is found in exon 1, c. $41 \mathrm{C}>\mathrm{A}$ (p.Pro14His) and within the 3 'UTR at c. ${ }^{\star} 82 \mathrm{C}>\mathrm{T}$, indicating the variant hotspots at either end of the gene in both familial and sporadic forms of schwannomatosis [11,12]. In contrast, the variants in the central exons or which delete all, or large parts, of the coding sequence often occurred in rhabdoid tumours. However, no exact genotype-phenotype correlation between variants in SMARCB1 has been found so far. Intriguingly, the deletion variant (c.885_896delGAAGCTGTGCTC p.(296_299del)) in our study located in the central exon (exon 7), caused familial schwannomatosis. To assess the phenotype-genotype relationship of SMARCB1, We evaluated the Human Gene Mutation Database (HGMD) regarding SMARCB1 variants and validated associated phenotype records. Overall, we found that about $51.1 \%$ $(69 / 135)$ of variants in SMARCB1 were located in Snf5 domain, $80.7 \%(109 / 135)$ of variants were loss-of- 
function (LOF) variants (Table 1). 44\% (59/134) and $26.2 \%(35 / 134)$ of variants were related to rhabdoid tumour and schwannomatosis separately (Table 2). We futher compared the variants in rhabdoid tumour and schwannomatosis, LOF variants are the main variant type in both diseases $\triangle$ Rhabdoid tumour VS schwannomatosis: 79.7\% (47/59) VS 82.9\% (29/35)) (Table 3), while more variants located in the Snf5 subunit of SMARCB1 in rhabdoid tumour $(67.8 \%, 40 / 59)$ than that in schwannomatosis $(25.7,9 / 35)$ (Table 2). These results indicated that LOF variants within the Snf5 subunit of SMARCB1 inclined to cause rhabdoid tumour.

Malignant peripheral nerve sheath tumors arising in schwannomatosis patients were reported in some cases [13]. But the prognosis on the schwannomatosis caused by SMARCB1 variant was uncertain. The

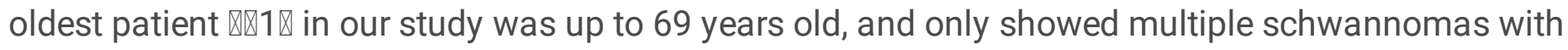
favorable outcome. Other patients (27 and 48 years-old, separately) in this pedigree has the similar symptoms, and need further follow-up.

Our study highlights the importance of genetic testing and counseling in patients with schwannomatosis, showed the consistent phenotype of the patients with the variant and expands the genetic background of schwannomatosis. Further and more studies of genotype and phenotype relationship are needed in order to better determine the cancer risk for carriers of SMARCB1 germline variants.

\section{Declarations}

\section{Acknowledgments}

We'd like to express our gratitude to all the participants who participated in this investigation. The authors would like to thank the patient's family for participating in this study specially.

\section{Authors' contributions}

$\mathrm{CW}$ contributed to the study conception and design. The manuscript was written by $\mathrm{CW}$ and YC. CW and $\mathrm{XZ}$ contributed to data retrieval and statistical analysis. Clinical data collection, genetic counseling and follow-up were performed by XK. WES analysis was performed by QL. CW performed Sanger sequencing. DNA was extracted by CC. CW, YC and CC modifed and proofread the paper. All authors read and approved the final manuscript.

\section{Funding}

This work was supported by National Natural Science Foundation of China (No.82000321), National Key Research and Development Program of China (No. 2018YFC1002206-2).

\section{Availability of data and materials}

The datasets used and analysed during the current study available from the corresponding author on reasonable request. 


\section{Ethics approval and consent to participate}

This study was approved by the Medical Ethics Committee of the First Affiliated Hospital of Zhengzhou University. Written informed consent was obtained from all participants.

\section{Consent for publication}

All patients in this study provided their consent for publication.

\section{Competing interests}

The authors declare that they have no conflict of interests.

\section{Author details}

${ }^{1}$ Genetics and Prenatal diagnosis Center, The First Affiliated Hospital of Zhengzhou University, Zhengzhou 450052, Henan, People's Republic of China. ${ }^{2}$ Department of Medical Genetics and Prenatal Diagnosis, Luoyang Maternal and Child Health Care Hospital, Luoyang 471000, Henan, People's Republic of China.

\section{References}

1. Kusafuka T, Miao J, Yoneda A, et al. Novel germ-line deletion of SNF5/INI1/SMARCB1 gene in neonate presenting with congenital malignant rhabdoid tumor of kidney and brain primitive neuroectodermal tumor. Genes Chromosomes Cancer. 2004,40(2):133-9. https://doi.org/10.1002/gcc.20026.

2. Plotkin SR, Wick A. Neurofibromatosis and Schwannomatosis. Semin Neurol. 2018,38(1):73-85. https://doi.org/10.1055/s-0038-1627471.

3. Smith MJ, Isidor B, Beetz C, et al. Mutations in LZTR1 add to the complex heterogeneity of schwannomatosis. Neurology. 2015,84(2):141-7. https://doi.org/10.1212/wnl.0000000000001129.

4. Kohashi K, Oda Y. Oncogenic roles of SMARCB1/INI1 and its deficient tumors. Cancer Sci. 2017,108(4):547-552. https://doi.org/10.1111/cas.13173.

5. Gonzalvo A, Fowler A, Cook RJ, et al. Schwannomatosis, sporadic schwannomatosis, and familial schwannomatosis: a surgical series with long-term follow-up. Clinical article. J Neurosurg. 2011,114(3):756-62. https://doi.org/10.3171/2010.8.JNS091900.

6. Smith MJ, Wallace AJ, Bowers NL, et al. Frequency of SMARCB1 mutations in familial and sporadic schwannomatosis. Neurogenetics. 2012,13(2):141-5. https://doi.org/10.1007/s10048-012-0319-8.

7. Rousseau G, Noguchi T, Bourdon V, et al. SMARCB1/INI1 germline mutations contribute to $10 \%$ of sporadic schwannomatosis. BMC Neurol. 2011,11:9. https://doi.org/10.1186/1471-2377-11-9.

8. Merker VL, Esparza S, Smith MJ, et al. Clinical features of schwannomatosis: a retrospective analysis of 87 patients. Oncologist. 2012,17(10):1317-22. 
https://doi.org/10.1634/theoncologist.2012-0162.

9. Agaimy A, Hartmann A, Antonescu CR, et al. SMARCB1 (INI-1)-deficient Sinonasal Carcinoma: A Series of 39 Cases Expanding the Morphologic and Clinicopathologic Spectrum of a Recently Described Entity. Am J Surg Pathol. 2017,41(4):458-71.

https://doi.org/10.1097/PAS.0000000000000797.

10. Masliah-Planchon J, Bièche I, Guinebretière JM, et al. SWI/SNF chromatin remodeling and human malignancies. Annu Rev Pathol. 2015,10:145-71. https://doi.org/10.1146/annurev-pathol-012414040445.

11. Smith MJ, Walker JA, Shen Y, et al. Expression of SMARCB1 (INI1) mutations in familial schwannomatosis. Hum Mol Genet. 2012,21(24):5239-45. https://doi.org/10.1093/hmg/dds370.

12. Boyd C, Smith MJ, Kluwe L, et al. Alterations in the SMARCB1 (INI1) tumor suppressor gene in familial schwannomatosis. Clin Genet. 2008,74(4):358-66. https://doi.org/10.1111/j.13990004.2008.01060.x.

13. Heatley N, Kolson Kokohaare E, Strauss DC, et al. Epithelioid malignant peripheral nerve sheath tumor arising in schwannoma. Rare Tumors. 2020,12:2036361320950862. https://doi.org/10.1177/2036361320950862.

\section{Tables}

Table 1 Variant types of SMARCB1

(HGMD® professinal 2021.2) 


\begin{tabular}{|c|c|c|c|}
\hline Variant Type & Total Number & $\begin{array}{l}\text { Varants in Snf } 5 \\
\text { domain }\end{array}$ & Resources \\
\hline Missense/Nonsense & 44 & 14 & HGMD \\
\hline Splicing & 20 & 7 & \\
\hline Regulatory & 3 & 0 & \\
\hline Small insertions & 12 & 5 & \\
\hline Small indels & 3 & 2 & \\
\hline Gross deletions & 25 & 22 & \\
\hline Gross insertions & 6 & 6 & \\
\hline Complex & 2 & 2 & \\
\hline Repeats & 0 & 0 & \\
\hline Small deletions+1 & $20+1$ & $11+1$ & HGMD+This report \\
\hline Total & $135+1$ & $69+1$ & \\
\hline
\end{tabular}

Table 2 Genotype-phenotype correlation of the variants in SMARCB1

(HGMD® professinal 2021.2) 


\begin{tabular}{|c|c|c|}
\hline Disease/phenotype & $\begin{array}{l}\text { Varants in Snf } 5 \\
\text { domain }\end{array}$ & $\begin{array}{l}\text { Number of } \\
\text { variants }\end{array}$ \\
\hline Rhabdoid tumour & 39 & 58 \\
\hline Rhabdoid tumour predisposition syndrome & 1 & 1 \\
\hline Schwannomatosis, familial & 5 & 22 \\
\hline Schwannomatosis & 4 & 13 \\
\hline Rhabdoid tumour predisposition \& schwannomatosis & 1 & 1 \\
\hline Coffin-Siris syndrome & 4 & 11 \\
\hline Atypical teratoid/rhabdoid tumours & 2 & 6 \\
\hline Brain and Kidney tumours & 2 & 3 \\
\hline CNS Atypical Teratoid/Rhabdoid tumours & 1 & 3 \\
\hline Rhabdoid predisposition syndrome & 2 & 2 \\
\hline Atrioventricular septum defects & 1 & 1 \\
\hline Atypical teratoid/rhabdoid tumour & 1 & 1 \\
\hline Cornelia de Lange syndrome & 1 & 1 \\
\hline Developmental delay and hypotonia & 0 & 1 \\
\hline Intellectual disability, severe syndromic & 0 & 1 \\
\hline Kidney tumours & 0 & 1 \\
\hline Kleefstra syndrome & 0 & 1 \\
\hline Lung adenocarcinoma & 0 & 1 \\
\hline Multiple congenital anomalies & 1 & 1 \\
\hline Multiple meningiomas & 0 & 1 \\
\hline Nicolaides-Baraitser syndrome & 0 & 1 \\
\hline Ovarian serous cystadenocarcinoma & 0 & 1 \\
\hline Posterior fossa brain tumours & 1 & 1 \\
\hline Ventricular septal defect & 0 & 1 \\
\hline TOTAL & 66 & 134 \\
\hline
\end{tabular}

Table 3 Variant types of SMARCB1 in Rhabdoid tumour VS Schwannomatosis. 
(HGMD® professinal 2021.2)

\begin{tabular}{llll}
$\begin{array}{l}\text { Rhabdoid } \\
\text { tumour }\end{array}$ & $\begin{array}{l}\text { Rhabdoid } \\
\text { tumour } \\
\text { predisposition } \\
\text { syndrome }\end{array}$ & $\begin{array}{l}\text { Schwannomatosis, } \\
\text { familial }\end{array}$ & Schwannomatosis \\
\hline 1 & 0 & 5 & 2 \\
\hline 0 & 0 & 1 & 1 \\
\hline 12 & 0 & 3 & 1 \\
\hline 4 & 1 & 8 & 3 \\
\hline 0 & 0 & 1 & 1 \\
\hline 6 & 0 & 2 & 1 \\
\hline 0 & 0 & 1 & 1 \\
\hline 21 & 0 & 0 & 0 \\
\hline 3 & 0 & 0 & 1 \\
\hline 1 & 0 & 0 & 0 \\
\hline 0 & 0 & 0 & 0 \\
\hline 10 & 0 & 0 & 13 \\
\hline 58 & 1 & 0 & \\
\hline
\end{tabular}

\section{Figures}




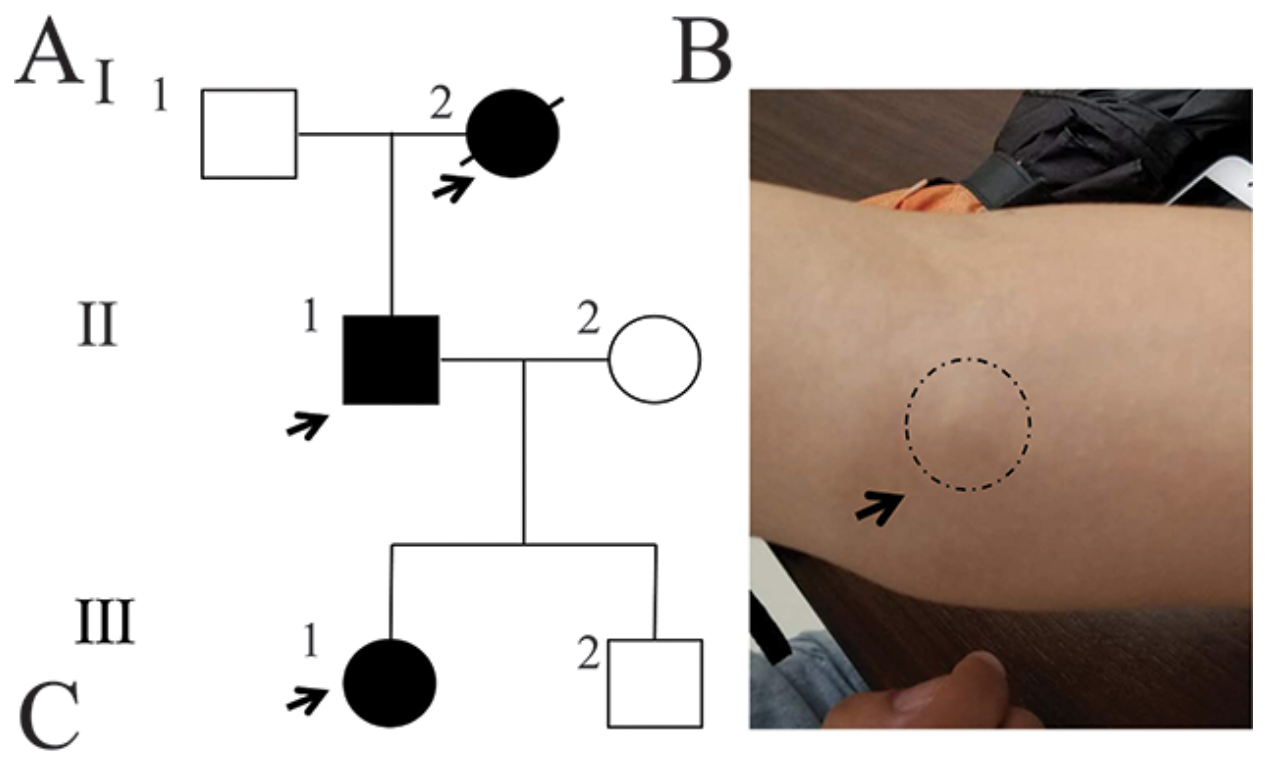

II1 SMARCB1 c.885_896delGAAGCTGTGCTC (p.295_299del)

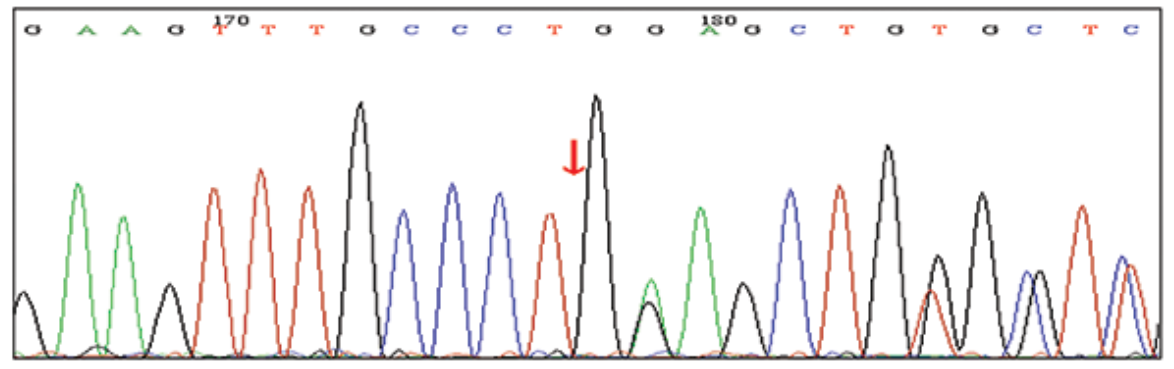

II2 SMARCB1 normal

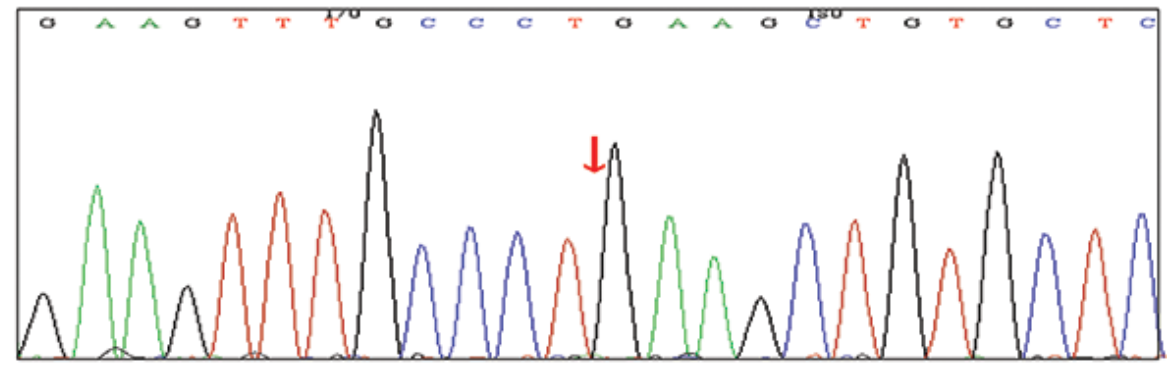

III1 SMARCB1 c.885_896delGAAGCTGTGCTC (p.295_299del)

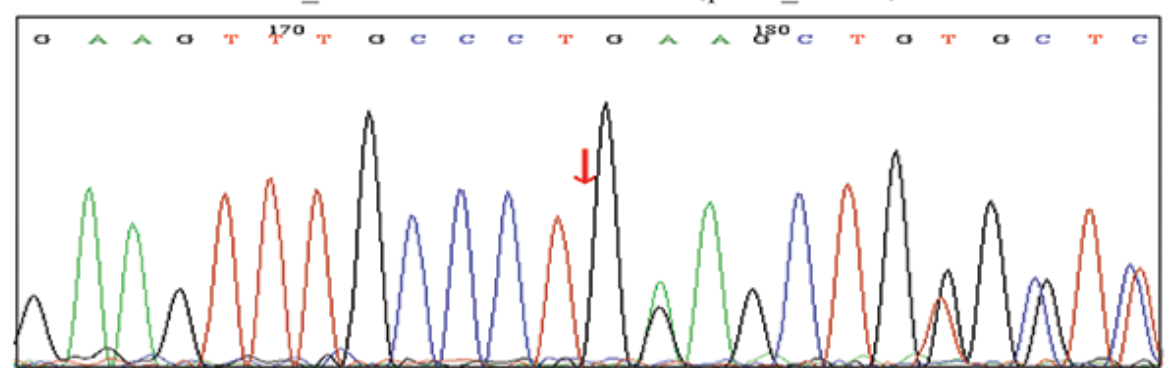

\section{Figure 1}

Pedigree analysis of the familial schwannomatosis patients. A: The simplified pedigree spanning 3 generations. Squares and circles denote males and females respectively. Filled squares or circles indicate probands (marked with the arrow), and the unfilled squares or circles are unaffected family members. B: Skin symptoms of schwannomatosis (skin lumps) are noted. C: Sequencing results of SMARCB1 of $₫ 1$, $\nabla 2$ and $\otimes 1$. 

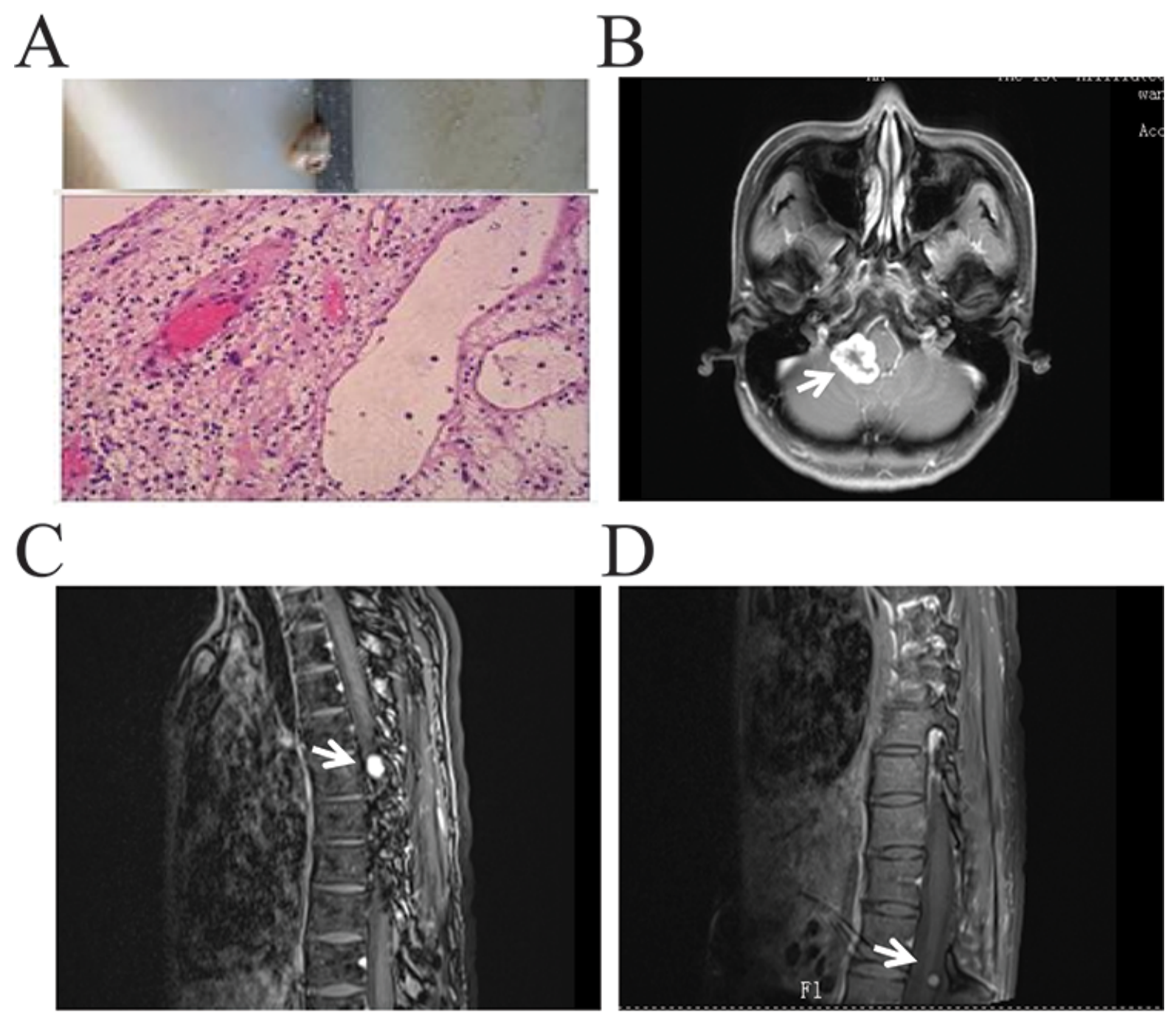

$\mathrm{E}$
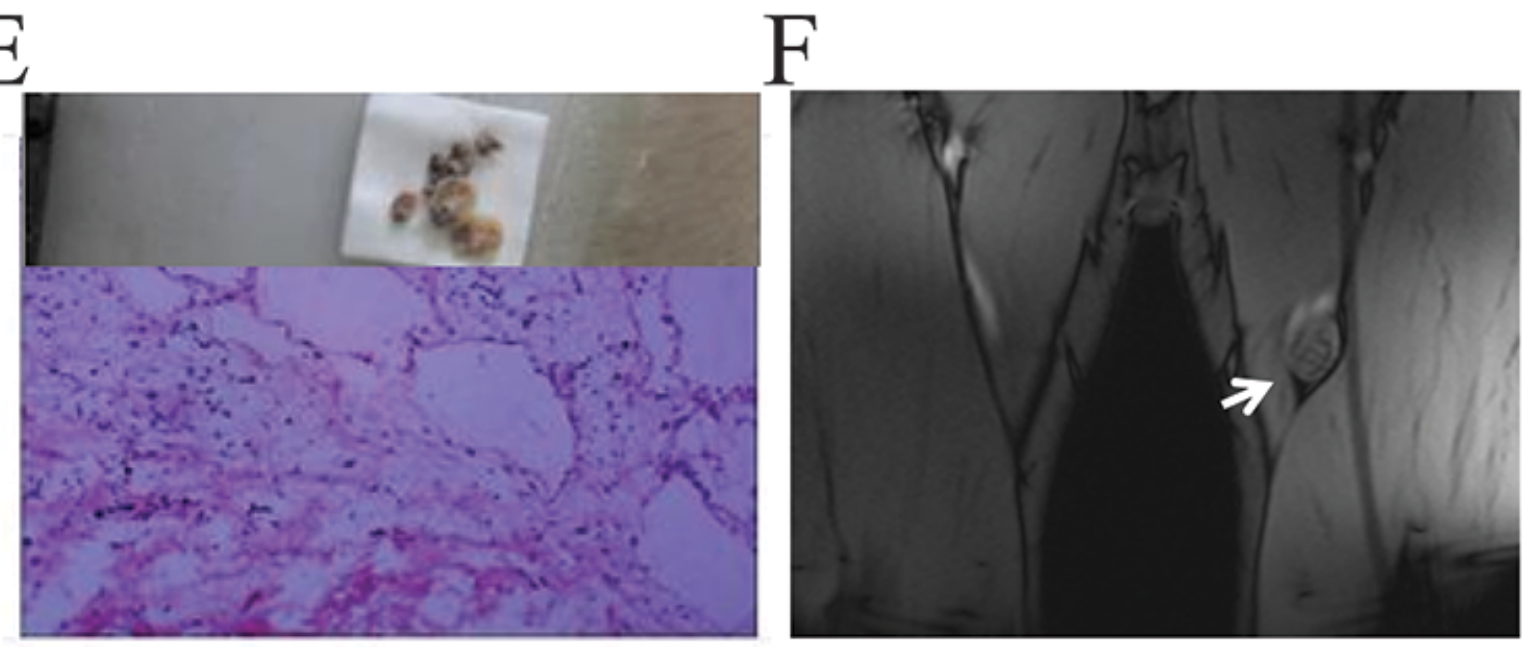

Figure 2

Pathological histology analysis and MRI tests of the familial schwannomatosis patients. A: Chest wall tumors of $₫ 1$ were resected and stained with Haematoxylin and eosin (H\&E). B-D: MRI tests demonstrating several benign intracranial, cervical intraspinal and thoracic intraspinal schwannomas (arrows). E: Tumors from the left leg of $₫ 1$ were resected and stained with Haematoxylin and eosin (H\&E). F: MRI tests demonstrating schwannomas within the left leg (arrows). 


\section{Supplementary Files}

This is a list of supplementary files associated with this preprint. Click to download.

- figs1.tif 